\title{
Dimension- and space-based intertrial effects in visual pop-out search: modulation by task demands for focal-attentional processing
}

\author{
Joseph Krummenacher · Hermann J. Müller • \\ Michael Zehetleitner · Thomas Geyer
}

Received: 13 February 2008 / Accepted: 22 September 2008 / Published online: 10 December 2008

(C) Springer-Verlag 2008

\begin{abstract}
Two experiments compared reaction times (RTs) in visual search for singleton feature targets defined, variably across trials, in either the color or the orientation dimension. Experiment 1 required observers to simply discern target presence versus absence (simple-detection task); Experiment 2 required them to respond to a detection-irrelevant form attribute of the target (compound-search task). Experiment 1 revealed a marked dimensional intertrial effect of $34 \mathrm{~ms}$ for an target defined in a changed versus a repeated dimension, and an intertrial target distance effect, with an 4-ms increase in RTs (per unit of distance) as the separation of the current relative to the preceding target increased. Conversely, in Experiment 2, the dimension change effect was markedly reduced $(11 \mathrm{~ms})$, while the intertrial target distance effect was markedly increased (11 ms per unit of distance). The results suggest that dimension change/repetition effects are modulated by the amount of attentional focusing required by the task, with space-based attention altering the integration of dimension-specific feature contrast signals at the level of the overall-saliency map.
\end{abstract}

J. Krummenacher $(\bowtie)$

Department of Psychology, University of Fribourg,

Rue de Faucigny 2, 1700 Fribourg, Switzerland

e-mail: joseph.krummenacher@unifr.ch

H. J. Müller · M. Zehetleitner · T. Geyer

General and Experimental Psychology,

Ludwig Maximilian University Munich, Munich, Germany

H. J. Müller

Birkbeck College, University of London, London, UK

\section{Introduction}

The present study is concerned with the influence of intertrial history on the performance of visual pop-out search tasks, depending on whether the response requires simple target detection, which may be performed preattentively, or further, focal-attentional processing of the target to extract some response-relevant attribute (which is different from the detection-relevant attribute). In particular, the question at issue is how the requirement for focal-attentional-that is, inherently spatial-processing influences intertrial effects that are thought to arise at a preattentive-that is, spatially parallel-stage of stimulus coding. Before developing this question in detail, it is useful to review (1) the core assumptions about the functional processing architecture made by current theories of visual search as well as the evidence for (2) intertrial effects in pop-out search and (3) their evident modulation by task factors. This sets the stage for the issue examined, namely how the intertrial effects are modulated by the relative demands for focal-attentional processing.

Functional architecture of visual search

Humans are continuously engaged in the (attentional) selection of information for the control of ongoing behavior. A prominent set of tasks to study selective attention in the visual domain has been the visual-search paradigm. In the typical search task, observers have to discern the presence (and sometimes further analyze response-critical attributes) of a target object presented in an array of nontarget, or distractor, objects. Numerous studies have shown that, while a target that differs from distractors by a unique feature (e.g., a single red circle within an array of green circles) can be detected efficiently and independently of the 
number of distractors in the display; search for a target defined by a conjunction of features (e.g., a red circle amongst red squares and green circles) is inefficient, with detection reaction times (RTs) increasing (reasonably) linearly as a function of the number of distractors. To explain this dichotomy in performance between feature and conjunction searches, Treisman and her colleagues (Treisman \& Gelade 1980; Treisman \& Sato 1990), in their feature integration theory (FIT), assumed that the selection of the target is achieved in a two-stage process. In the first (parallel) stage, a limited set of basic object features (such as red and green, or circle and square) are coded and represented, in parallel across the field, in multiple (topographic) maps of feature analyzers, which are dimensionally organized (e.g., color maps, form maps). In the second (serial) stage, the separately coded features of an object are bound into a coherent object representation by a focal-attentional mechanism that can be allocated to only one object at the time (i.e., focal attention gates the featural information available at a particular location to an object recognition system, which represents the features bound into a coherent whole). FIT proposes essentially that, when the task requires bound-feature information to discern target presence, then search is limited by the serially operating focal-attention mechanism. In contrast, when the target is singled out by a unique feature, its presence can be established based on the output of the initial parallel stage of processing.

The guided search (GS) theory proposed by Wolfe and his colleagues (e.g., Wolfe, Cave, \& Franzel 1989; Cave \& Wolfe 1990; Wolfe 1994) maintains the two-stage architecture envisaged by FIT, but proposes that preattentive processing mechanisms help to guide focal attention to likely candidate targets amongst the display objects, and that only those that achieve high 'overall saliency' are selected for further processing by focal attention. Overall-saliency is represented by a master map of locations, which receives input from the topographically arranged feature analyzers that register, independently and in parallel, the color, form, etc., of the objects present in the field. The feature values thus coded for a given object are compared within dimensions (e.g., color) with those of surrounding objects, and the resulting dimension-specific feature contrast or saliency signals are then integrated across dimensions by the supradimensional master map (or overall-saliency) units. Focal attention is then allocated to that location, which achieves the highest saliency at the master map level. On this account, when the target is uniquely defined by a conjunction of features, the overall-saliency signal generated by the target differs relatively little from the signals produced by the various distractors and, assuming noise in the saliency computation process, some of the distractors may generate stronger overall-saliency signals, which then have to be serially inspected by focal attention before the target is eventually detected (serial search). This is in contrast to when the target is defined by a unique feature, in which case the target achieves a high overall-saliency value relative to the distractors, guiding focal attention directly to its location (parallel search).

Intertrial effects in visual search for pop-out targets

While GS has been very successful in accounting for search performance in tasks in which the target-defining feature(s) are fixed and known, it encountered a difficulty in explaining the detection of singleton feature targets whose defining features were variable across trials-in particular, when the target-defining dimension varied randomly across trials, for example, when the target could be unique in color on one trial and unique in shape (or orientation) on the next. Müller, Heller, and Ziegler (1995) (see also Treisman 1988) observed that, while search continued to operate in parallel across the field in such situations (i.e., there were no effects of the number of items in the display), RTs to the very same target, such as the only red item amongst green distractors, were overall increased in crossdimension search (when the target dimension was variable across trials) compared to intra-dimension search (when the target defining feature was variable within a fixed dimension, e.g., sometimes red and sometimes blue). Subsequently, Found and Müller (1996) found that, in crossdimension search, target detection is expedited when the current target $N$ is defined in the same dimension as the preceding target $N-1$ (no matter whether successive targets are featurally the same or different) compared to when it is defined in a different dimension. Müller and his colleagues took this pattern of a cross-dimension (vs. intradimension) search cost and an intertrial dimensional change (vs. repetition) cost (in cross-dimension search) to argue for what they termed a 'dimension weighting' (DW) account [for recent overviews, see Müller, Reimann, and Krummenacher (2003) and Müller and Krummenacher (2006)], that is, within a GS-type processing architecture, dimension-specific feature contrast signals are attentionally weighted (or amplified) prior to their integration by master map units, with the total weight being limited such that upmodulating the weight for one dimension entails downmodulating the weight for other dimensions [see Zehetleitner and Müller (2008)]. Importantly, the DW account assumes that dimension-specific weighting effects arise at an early, preattentive stage of perceptual processing (consistent with related accounts, such as the contingent-attentional capture hypothesis of Folk and his colleagues, e.g., Folk, Remington, \& Johnston 1992) - that is, a stage prior to the allocation of focal attention, which in turn provides the gateway to post-selective stimulus analysis and response selection processes. 


\section{Modulation of intertrial effects by task factors}

However, this core assumption of the DW account has recently been challenged by a number of studies that have reexamined dimension-based effects and came to the conclusion that the 'weighting' effects described by Müller and his colleagues (Müller et al. 1995; Found \& Müller 1996) arise at a late, postselective stage of processing (following focal-attentional selection), at which detected targets are translated into responses (e.g., Cohen \& Magen 1999; Mortier, Theeuwes, \& Starreveld 2005; Theeuwes, Reimann, $\&$ Mortier 2006). This challenge has been based in part on findings in so-called cross-dimensional 'compound-search' tasks, where the detection-relevant attribute of the target (e.g., its unique color) is independent of the responserelevant attribute (e.g., the target's form). In such tasks, dimension-specific intertrial effects are typically reduced overall, sometimes toward nonsignificant levels, relative to simple detection tasks [e.g., 6 vs. $35 \mathrm{~ms}$ in Krummenacher, Müller, and Heller (2002b); 10 vs. $34 \mathrm{~ms}$ in Theeuwes et al. (2006); 4 vs. 48 ms in Pollmann, Weidner, Müller, and von Cramon (2000, 2006), respectively]. The argument against the DW account is that, if the weighting effects indeed arose at a preattentive stage of processing, then the effect of a dimension change from trial $N-1$ to trial $N$ should be equivalent in both tasks, as both tasks involve the same preattentive coding processes. However, given that a change in response requirements alters this pattern, this must mean that the dimension change effect in simple detection tasks arises at a postselective, response-related level (e.g., biasing response selection towards repeating the last response).

However, in a meta-analysis of a number of compoundtask studies, Müller and Krummenacher (2006) have recently found that the preattentive (saliency) coding and postselective (target analysis and response selection) processes are not operating in a strictly additive fashion in compound tasks, as assumed by the postselective accounts. Rather, there was an interaction between repetition/change of the target-defining dimension on the one hand and repetition/change of the response (or response-relevant attribute) on the other hand: A significant dimension change effect was evident only when the response remained the same, in which case compound-task RTs were expedited for a repetition versus a change of the target-defining dimension; in contrast, when the response changed, this effect was either abolished or tended to be reversed (with slightly faster RTs for a change vs. a repetition of the target defining dimension). [Consequently, as a result of collapsing the data over response repetition and change conditions, the overall magnitude of the dimension-specific intertrial effects is greatly reduced, if not completely abolished.] Müller and Krummenacher (2006) proposed that this pattern of effects reflects (implicit) linkages between stimulus and response 'expectancies' [along the lines of Kingstone (1992)], such that a repetition of the target-defining dimension is associated with a repeated response, and a change in the target dimension with a changed response. Kingstone (1992) showed that such linkages may be assumed by the system even if stimulus and response events are uncorrelated [as in the studies reviewed by Müller and Krummenacher (2006)] or negatively correlated. Consequently, in terms of stimulus-response (S-R) translation, deciding on the same response as on the preceding trial would be expedited if the target-defining dimension is repeated, and slowed if the target-defining dimension changes. However, focal-attentional selection of the target (which precedes S-R translation) should be expedited if the target dimension is repeated rather than changed, irrespectively of whether the response is repeated or changed.

This reasoning has recently been examined in an ERP (event-related potential) study by Töllner, Gramann, Müller, Kiss and Eimer (2008). The logic of this study was to 'decompose' the behavioral response times in terms of the underlying preattentive coding, focal-attentional selection, stimulus-response translation, and response production processes. As a marker of the transition between preattentive processing and focal-attentional processing, the N2pc component was analyzed. The N2pc is a negative-going deflection with a maximum over visual areas of the hemisphere contralateral to the location of an attended stimulus, which is interpreted as reflecting the attentional selection of target among nontarget stimuli, based on target-defining perceptual attributes (e.g., Eimer 1996; Woodman \& Luck 1999; Hopf, Boelmans, Schoenfeld, Heinze, \& Luck 2002). Importantly, as predicted, Töllner et al. found the N2pc to be elicited faster, and be larger in amplitude, when the target-defining dimension was repeated, rather than changed, between trials, independently of whether the response was repeated or changed.

Besides the N2pc, Töllner et al. analyzed the LRP (lateralized readiness potential). The LRP is typically observed over the motor area contralateral to the side of a unimanual response, and is thought to mark the start of effector-specific response activation and execution processes that occur after response selection has been completed. Importantly, Töllner et al. found the response-locked LRPs (indicative of response activation and execution) to be differentially affected by response repetitions versus alternations between trials, but not by dimension repetitions versus alternations-the latter is as predicted by the DW account. In addition, Töllner et al. analyzed stimulus-locked LRPs to further examine how dimension and response changes versus repetitions affect processing stages that precede response activation and execution. Because stimuluslocked LRP latencies are determined both by the time it 
takes to attentionally select and analyze the target and by the time required to select an appropriate response, these latencies may provide insight into the time demands of response selection processes that are intermediate between attentional target selection (indexed by the N2pc) and response production (indexed by the response-locked LRP). The results revealed that these intermediate processes are indeed shorter when both the target-defining dimension and response are repeated and when both are changed; but longer when one is repeated, but the other changed. This is exactly the pattern predicted by Müller and Krummenacher's (2006) account in terms of linked stimulus and response expectancies (see above).

Influence of the relative task demands for focal-attentional processing

The present study was designed to examine another factor, besides response repetition/change, that potentially also modulates dimension repetition effects in compound-search tasks, but not (or much less so) simple detection tasks, namely, spatial attention. In simple-detection tasks, detection of an above-threshold overall-saliency signal is sufficient to permit a target-present response, that is, responding does not require the allocation of focal attention to the target [for discussion, see Krummenacher et al. (2002b) and Müller and Krummenacher (2006)]. Accordingly, saliency signal computation would operate in an alike manner in parallel across the field, without involving any spatialattentional modulations (biases). That is, performance should be relatively uninfluenced by the positioning of the target on trial $N$ relative to that of the target on trial $N-1$. By contrast, in compound tasks, detection of an abovethreshold overall-saliency signal is insufficient to permit a response to be triggered. Rather, focal attention needs to be allocated to the target location so as to further process the target to permit the response-relevant attribute to be discriminated and a corresponding response to be issued. Conceptually, the required focusing of attention could have two effects as defined in the following paragraphs.

First, the need for focused attention in compound tasks may introduce larger positional repetition/change effects, as observed in tasks where the critical target feature within a fixed dimension could vary randomly across trials (e.g., Maljkovic \& Nakayama 1996; Geyer, Müller, \& Krummenacher 2007). In particular, there has been a marked bias on trial $N$ to reallocate focal attention to the target location on trial $N-1$, expediting the compoundtask response when the target actually reappeared at this location, rather than at a previous distractor or empty location (and this bias was independent of whether or not there was a change in the target-defining feature; Maljkovic \& Nakayama 1996; Geyer \& Müller 2008a). This location-based intertrial effect may be interpreted as reflecting a spacebased (as opposed to dimension- or feature-based) weighting mechanism that is likely to operate on the overall-saliency map, weighting the incoming (dimensions-specific feature contrast) signals as a function of their distance from the previous target location. Effectively, the result is that activity build-up (responsivity) on the overall-saliency map is faster (greater) for repeated, compared to changed, target positions. If the space-based weighting, operating within the overall-saliency map, is independent of the dimensionbased weighting, which operates on the incoming signals to the overall-saliency map, then this would predict an interaction such that the dimension-based effect should be larger for target locations on trial $N$ near the target location on trial $N-1$, as compared to locations further away-because the same difference in 'bottom-up' signals between dimension repetition and change trials would be amplified more for near as compared to far locations.

Note that the spatial separation of the target $N$ relative to that of target $N-1$ may also interact with response repetition/change, as a larger distance would violate the 'expectancy' of the target reoccurring at approximately the same position as that on the previous trial.

Second, assuming that the dimensional and spatial weights are, in some sense, drawn from the same (limited) pool of attentional weights [though see Maljkovic and Nakayama (1996), who showed positional repetition effects to be independent of featural repetition effects], then the stronger engagement of spatial weighting in compound, as compared to simple-detection, tasks might interfere with dimensional weighting and thus diminish any effects resulting from the repetition (vs. change) of the target-defining dimension. As a result, repetition/change effects associated with target position should be larger in compound-search relative to simple-detection tasks, whereas dimension repetition/change effects should be larger in detection than in compound tasks.

As the DW account in its present form is agnostic as to effects of spatial weighting (but see Krummenacher et al. 2002a), the aim of the present study was twofold: to systematically examine, in a comparison of a simple detection versus a compound-search tasks, (1) how any space-based effects would be topographically characterized in the two tasks and (2) at which processing stage spatial weighting processes are likely to modulate dimensional weighting. To do so, in Experiment 1, observers had to simply decide on the presence (vs. absence) of an odd-one-out color or orientation item in the display. In Experiment 2, following detection of the target item, observers were additionally required to discern the response-relevant attribute-the location of a line placed randomly at the top or bottom of the target (note that lines were also positioned at the top or bottom of distractors). To examine changes (repetitions) of the target 
Fig. 1 Example of the search displays presented in (a) the singleton detection task of Experiment 1 (orientation target trial), and (b) the compound task of Experiment 2 (color target trial). (Note that search items and distances are not drawn to scale.) a)

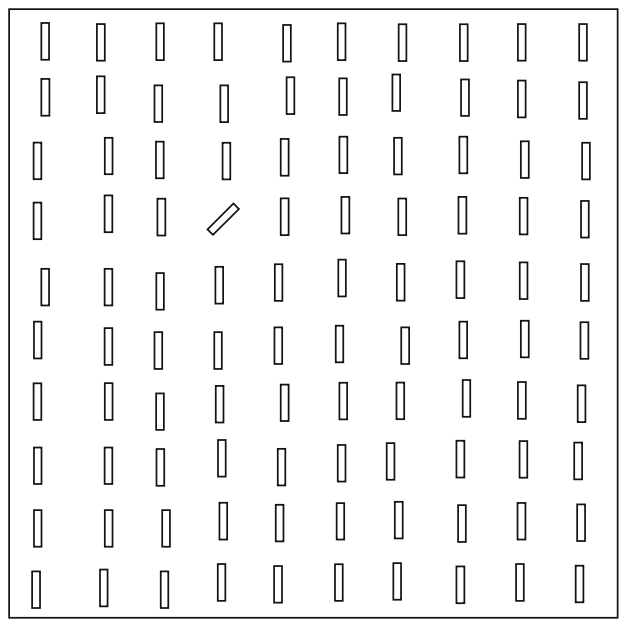

b)

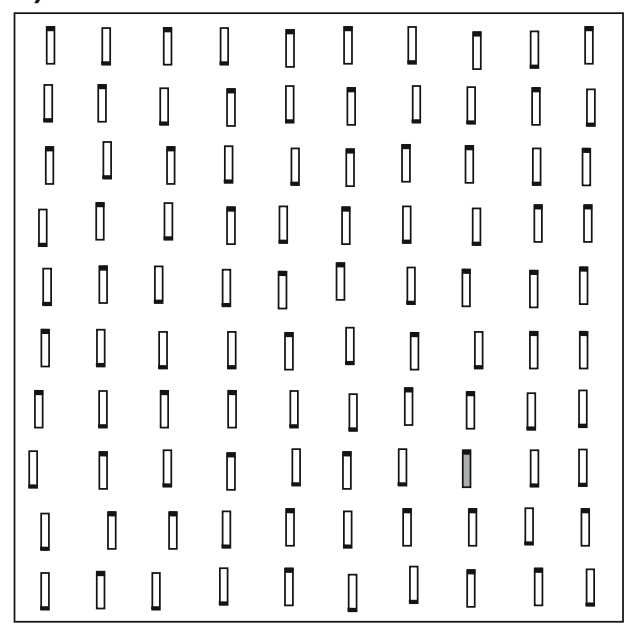

location across trials, the search display comprised an array of $10 \times 10$ items, with targets appearing in the inner $8 \times 8$ matrix locations. Thus, target location changes could be examined for distances (in the following abbreviated by the letter $d$ ) of up to seven cells of the virtual matrix underlying the search displays.

\section{Experiment 1}

\section{Method}

\section{Apparatus, task, and stimuli}

Experiment 1 examined the relation of dimension- and position-based intertrial effects in a singleton feature search (detection) task. Observers were instructed to indicate, as quickly and as accurately as possible, the presence versus absence of a red vertical (color-defined) or green 45-degree right-tilted (orientation-defined) target bar among a homogeneous set of green vertical distractor bars (see Fig. 1a, lefthand panel, for an illustration of the search display). The bars were $1.25^{\circ}$ of visual angle in height and $0.25^{\circ}$ in width, were isoluminant (red: VGA RGB $255,0,0 ; 3.4 \mathrm{~cd} / \mathrm{m}^{2}$; green: RGB 0, 248, $0 ; 3.4 \mathrm{~cd} / \mathrm{m}^{2}$; black screen background), and were placed in the cells of a virtual $10 \times 10\left(20^{\circ} \times 20^{\circ}\right)$ matrix, with item positions randomly jittered vertically and horizontally by up to $0.25^{\circ}$ relative to the cell center (so that the interitem distances ranged between $1.5^{\circ}$ and $2.5^{\circ}$, with an average of $2^{\circ}$ ). Presentation of target items was restricted to the inner $8 \times 8$ matrix positions, so as to avoid edge effects (observers were not informed about this).

Stimulus presentation, timing, and response recording were controlled by a Pentium PC running under the DOS operating system. Search displays were presented on a $19^{\prime \prime}$ CRT monitor. Observers viewed the monitor from a dis- tance of $60 \mathrm{~cm}$, with distance maintained by the use of a chin rest. They responded 'target-present' and 'targetabsent' by pressing the right and left buttons, respectively, of a serial Microsoft mouse, with track ball removed to improve timing accuracy.

\section{Procedure and timing}

Each trial started with the presentation of a central fixation marker (for $500 \mathrm{~ms}$ ), followed by the onset of the search display. On target-present trials, one of the green vertical distractors was replaced by either a color- or an orientationdefined target. In each block, target-absent (green vertical bars only) and target-present trials were equally frequent; $50 \%$ of the latter trials contained a color target (red vertical bar), and $50 \%$ and orientation target (green $45^{\circ}$ right-tilted bar). The various trial types were presented in randomized order within blocks. The search display remained visible until observers responded. After the response, a black screen appeared for an intertrial interval of 1,000 ms. Error feedback was provided by a brief computer-generated tone $(880 \mathrm{~Hz})$; no feedback was given after a correct response.

The entire experiment comprised two sessions (each of about $25 \mathrm{~min}$, with a 5 -min break between sessions), each of 20 blocks of 52 trials, for a total of 2,080 trials. Observers were free to take breaks between experimental blocks. Prior to the experiment, observers practiced the task by completing at least one block of 52 trials, and they decided to start the experiment when they felt comfortable with the task.

\section{Participants}

Ten observers participated in Experiment 1 (five female; age range 20-28 years, median age 23 years). All had either normal or corrected-to-normal vision and all reported normal color vision. Observers were paid at a rate of $€ 6.00$ per 
hour or received course credits. All the observers were naïve as to the purpose of the experiment, and none of them had previous experience with visual search tasks.

Results and discussion

Error rates were low overall (4.62\%), with equal proportions of false alarms (4.40\%) and misses (4.84\%) $[t(9)=-0.588$, n.s. $]$. Besides error trials, correct-response trials with reaction times (RTs) shorter than $200 \mathrm{~ms}$ ('anticipations') and longer than 1,200 ms ('failures to respond in time'), overall less than $5 \%$ of the data, were excluded from analysis. Furthermore, trials with RTs exceeding the individual mean RT by more or less than three standard deviations (SD) were excluded as outliers, for each experimental condition (target-absent, color target, orientation target; less than $1.5 \%$ of all remaining trials).

Target-present RTs were subjected to a three-way repeated-measures ANOVA with the factors target dimension (color, orientation), dimensional intertrial transition (same dimension, different dimension), and intertrial target distance $(\mathrm{d} 0, \mathrm{~d} 1, \ldots, \mathrm{d} 7)$. This ANOVA revealed only the three main effects to be significant: RTs were faster for color targets compared to orientation targets [434.7 vs. $460.3 \mathrm{~ms}, \quad F(1,9)=60.923 ; \quad \mathrm{MSe}=859.105 ; \quad P<0.001]$; faster for repeated-dimension targets compared to changeddimension-targets $[430.8$ vs. $464.3 \mathrm{~ms}, F(1,9)=49.994$; MSe $=1,794.372 ; P<.001]$; and RTs increased significantly with increasing distance of the target locations on consecutive trials $[F(4.269,38.421)=9.817 ; \mathrm{MSe}=699.509$; $P<0.05$ (Huyhn-Feldt-corrected $d f$ )].

None of the interactions was significant [target dimension $\times$ dimensional intertrial transition, $F(1,9)=2.966$, $\mathrm{MSe}=193.736, P>0.1$; target dimension $\times$ intertrial target distance, $F(7,63)<1$, n.s.; dimensional intertrial transition $\times$ intertrial target distance, $F(4.387,39.484)<1$, n.s., Huyhn-Feldt-corrected $d f$; dimension $\times$ dimensional intertrial transition $\times$ distance, $F(7,63)=1.120, \quad$ MSe $=$ $262.894, P>0.35]$. The dimension-based intertrial transition effect of $33.5 \mathrm{~ms}$ (see Fig. 2) is within the 35- to 40-ms range reported in earlier studies (e.g., Found \& Müller 1996).

Theoretically of most interest in the present context is the effect of intertrial target distance. Table 1 (top panel) presents RT as a function of the distance, in units of cells of the virtual display grid, between the target location on the current trial $N$ relative to that on the preceding trial $N-1$ (see also Fig. 2). The difference between the minimum (d0, i.e., location repetition) and maximum shift (d7) was $28.6 \mathrm{~ms}$. Planned comparisons (see corresponding $F$ and $P$ values in Table 1) of the RT differences for target shifts of $\mathrm{d} 1$ to $\mathrm{d} 7$ distance units relative to location repetitions (d0) revealed shifts larger than three units (d3), and particularly those equal to and larger than four units (d4), to yield

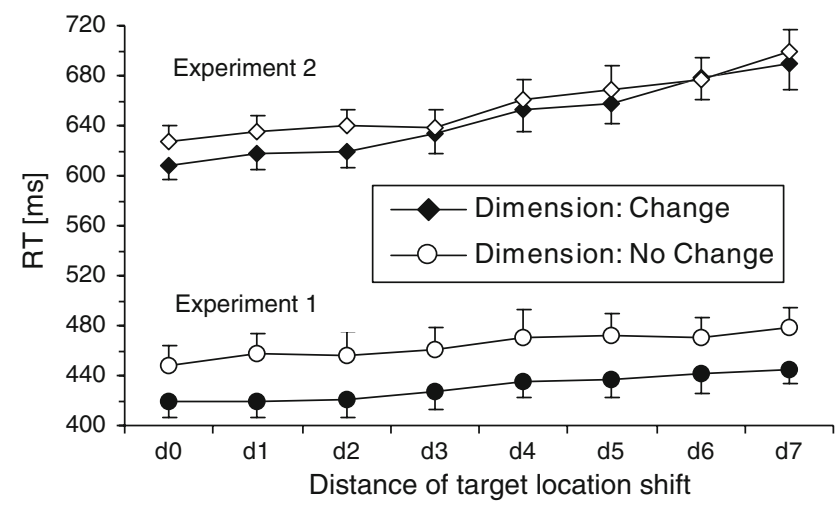

Fig. 2 Mean RTs in the detection task of Experiment 1 (circles, lower half of the panel) and compound task of Experiment 2 (diamonds) separately for dimension repetition (black markers) and dimension change trials (white markers). (Error bars indicate standard error of the mean.) Further, RTs are shown as a function of distance (d0 to d7) between target locations in consecutive trials $(N-1 \rightarrow N)$. A distance unit of 0 indicates target location repetitions, distance units of 1-7 refer to target location shifts by $1-7$ cells in the stimulus matrix

significant RT costs; no costs were produced by shifts of one (d1) and two (d2) units. Despite showing little increase in RT for small distance shifts, overall the distance effect is reasonably linear (linear regression: $R^{2}=0.961, P<0.01$ ), with an increase of $4.1 \mathrm{~ms}$ per unit of distance.

In summary, Experiment 1 showed that, in singleton feature detection tasks, the effect of changing the target location across trials ( $4 \mathrm{~ms}$ per unit of distance) is relatively small compared to, and independent of, the effect of changing the target-defining dimension $(30-40 \mathrm{~ms})$. It is possible that this shallow distance effect is due to the low, or absent, requirements for focal-attentional target analysis in singleton feature detection tasks (see 'Introduction'). If so, stronger intertrial location shift effects (perhaps coupled with reduced dimension-specific effects) may be observed in compound tasks, which make increased demands on focal-attentional processing. Furthermore, since target- and response-defining attributes vary independently in compound tasks, effects of dimensional and, possibly, spatial variations of the targets on consecutive trials may be interacting with effects of response changes and repetitions (e.g., Müller \& Krummenacher 2006; Töllner et al. 2008). Experiment 2 used a compound task to examine these possibilities.

\section{Experiment 2}

Method

Task, stimuli, and procedure

Experiment 2 was similar in method to Experiment 1, except that a compound-search task was used instead of a 
Table 1 Mean RTs for target location repetitions (d0) and shifts by 1-7 units of distance (d1-d7, with distances equivalent to single cells of a virtual matrix underlying the search array), separately for Experiments 1 and 2

\begin{tabular}{|c|c|c|c|c|c|c|c|c|}
\hline & \multicolumn{8}{|c|}{ Distance } \\
\hline & d0 & $\mathrm{d} 1$ & $\mathrm{~d} 2$ & $\mathrm{~d} 3$ & $\mathrm{~d} 4$ & d5 & d6 & $\mathrm{d} 7$ \\
\hline \multicolumn{9}{|l|}{ Experiment 1} \\
\hline Mean RTs (ms) & 433.1 & 438.7 & 438.9 & 443.6 & 453.3 & 454.7 & 456.1 & 461.7 \\
\hline $\mathrm{RT}$ costs (ms) & & 5.6 & 5.8 & 10.4 & 20.1 & 21.5 & 23.0 & 28.6 \\
\hline \multicolumn{9}{|l|}{ Planned contrasts } \\
\hline$F(1,9)$ & & 1.25 & 1.38 & 5.63 & 25.14 & 27.32 & 28.33 & 14.22 \\
\hline$P($ exact $)$ & & 0.292 & 0.270 & 0.042 & 0.001 & 0.001 & 0.000 & 0.004 \\
\hline \multicolumn{9}{|l|}{ Experiment 2} \\
\hline Mean RTs (ms) & 618.1 & 626.4 & 629.0 & 635.9 & 656.4 & 663.6 & 677.5 & 694.4 \\
\hline $\mathrm{RT}$ costs (ms) & & 8.2 & 10.9 & 17.7 & 38.2 & 45.5 & 59.4 & 76.2 \\
\hline \multicolumn{9}{|l|}{ Planned contrasts } \\
\hline$F(1,9)$ & & 6.43 & 21.25 & 12.05 & 64.24 & 42.52 & 59.37 & 56.47 \\
\hline$P($ exact $)$ & & 0.032 & 0.001 & 0.007 & 0.000 & 0.000 & 0.000 & 0.000 \\
\hline
\end{tabular}

RT costs indicate the difference between location repetitions and shifting the target by d units. Planned comparison ANOVA parameters $F$ and $P$ reveal statistical significance of RT costs associated with target location shifts

simple detection task and a target was present on all trials. The stimuli and their spatial arrangement (as well as the timing on each trial) were the same as in Experiment 1, with the search-critical features differentiating the target from distractors (green vertical bars) being either its color (red vertical bar) or its orientation (green $45^{\circ}$ right-tilted bar). The response-critical feature consisted of a white line presented either at the top (randomly selected $50 \%$ of stimuli in the display) or at the bottom edge (50\% of stimuli) of each bar stimulus, whether distractor or target (see Fig. 1b, right-hand panel, for an illustration). The bars were $1.25^{\circ}$ high and $0.25^{\circ}$ wide (as in Experiment 1); the additional white line, presented superimposed on the top or bottom edge of the each bar, was $0.1^{\circ}$ high and $0.25^{\circ}$ wide. Observers' task was to detect (and localize) the singleton feature target and respond according to the location, top versus bottom, of the response-relevant white line by pressing the right (top) or left (bottom) mouse button, respectively.

The experiment itself consisted of 16 blocks of 64 trials (1.024 experimental trials in total, taking less than $1 \mathrm{~h}$ to complete), performed in two consecutive sessions separated by a break of at least $5 \mathrm{~min}$. Prior to the experiment, observers completed at least one block of 56 practice trials to become familiar with the task.

\section{Participants}

Ten new observers took part in Experiment 2 (six females; age range 22-31 years; median age 25 years; normal or corrected-to-normal vision, including color vision). All observers were naive as to the purpose of the experiment, and none of them had previous experience with visual search tasks.
Results and discussion

Error rates were very low overall $(1.75 \%)$, with significantly higher rates for color- than for orientation-defined targets [2.74 vs. $0.75 \%$; two-tailed $t$ test: $t(9)=3.6435$; $P<0.05]$. Error trial RTs, RTs outside the range 200 $1,200 \mathrm{~ms}(0.9 \%$ of all trials), and, additionally, RTs outside three standard deviations of each observer's mean RT (1.1\% of all trials) were excluded from further analysis.

RT data were subjected to a five-way repeated-measures ANOVA with the factors target dimension (color, orientation), dimensional intertrial transition (dimension repetition, dimension change), response attribute (left hand, right hand), intertrial response transition (same response, different response), and intertrial target distance ( $\mathrm{d} 0, \mathrm{~d} 1, \ldots, \mathrm{d} 7)$. Overall mean RT was at $650.2 \mathrm{~ms}$. There was a tendency toward faster responses on trials with orientation compared to color targets (11.6-ms effect: 644.4 vs. $656.0 \mathrm{~ms}$; main effect of target dimension: $F(1,9)=3.206 ; \quad \mathrm{MSe}=$ $13,441.204 ; P=.107)$. Trials on which the target dimension was repeated showed a small, but highly significant RT benefit relative to trials with a dimension change [11.1-ms effect: 644.6 vs. $655.7 \mathrm{~ms}$; main effect of dimensional intertrial transition: $F(1,9)=92.081 ; \mathrm{MSe}=425.012 ; P<0.001]$. Right-hand responses were faster than left-hand responses [641.4 vs. $658.9 \mathrm{~ms}$; main effect of response attribute: $F(1,9)=5.683 ; \mathrm{MSe}=17,094.934 ; P<0.05]$, reflecting the fact that eight out of the ten participants were right-handed. Overall RTs tended to be faster for response repetition than for response change trials [11.9-ms effect: 644.2 vs. $656.1 \mathrm{~ms}$; main effect of intertrial response transition: $F(1,9)=5.042 ; \mathrm{MSe}=9,031.424 ; P>0.05]$. Finally, shifts 
in the target location from the previous to the current trial highly significantly affected RTs [main effect of intertrial target distance: $F(2.113,19.021)=44.725 ; \mathrm{MSe}=8,738.709$; $P<0.001$ (Huyhn-Feldt-corrected $d f$ )].

In contrast to Experiment 1 (singleton feature detection task), RTs tended to be faster to orientation-defined targets compared to color-defined targets in the compound task of Experiment 2 (reversing the pattern of effects evident in the detection task). Although this effect was not significant, it may be taken to suggest an overall increase in the weight assigned to the orientation (form) dimension throughout the experiment, probably because a form attribute of the target was consistently response-relevant (see Krummenacher, Müller, and Heller 2002b).

Analogous to Experiment 1, the effects of changing the target location across trials was examined further by planned comparisons of target location shift trials (distances $\mathrm{d} 1$ to $\mathrm{d} 7$ ) relative to location repetition trials (d0). In contrast to the pattern observed in Experiment 1 (detection task), RTs in the compound task were significantly slower for all shift distances (d1 to d7) relative to location repetitions (see Table 1, bottom panel, and Fig. 2). Despite a tendency of an accelerated increase in RT for large distance shifts, overall, the distance effect is reasonably linear (linear regression: $\left.R^{2}=0.962, P<0.001\right)$, with an increase of $10.9 \mathrm{~ms}$ per unit of distance.

Of the two-way interactions, only two were significant: intertrial dimension transition $\times$ intertrial response transition $[F(1,9)=101.238 ; \mathrm{MSe}=1,777.332 ; P<0.001]$ and, interestingly, intertrial response transition $\times$ intertrial target distance $[F(1,9)=7.852 ; \mathrm{MSe}=1,380.159 ; P<0.001]$. The former interaction arose because, on dimension repetition trials, changing the response attribute was associated with RT costs of $35.6 \mathrm{~ms}$. In contrast, there were no such costs on dimension change trials; instead, RTs were $12.2 \mathrm{~ms}$ faster [two-tailed paired-samples $t(9)=4.002$, $P<0.01]$ on trials on which both the target-defining and the response-relevant attribute changed, compared to trials on which only one of the two attributes changed (see Fig. 3).

The interaction of intertrial dimension change $x$ intertrial response change was modified by the response attribute $[F(1,9)=10.559 ; \mathrm{MSe}=159.591 ; P=0.010]$, owing to a slightly larger RT gain on trials requiring a left-hand relative to a right-hand response when both the target and response attributes changed (gain of $15.4 \mathrm{~ms}$ ) compared to when only the response attribute, but not the target attribute, changed (gain of $9.9 \mathrm{~ms}$ )

Further, the interaction between intertrial response transition $\times$ intertrial target distance was qualified by dimensional intertrial transition $[F(7,63)=2.232$; $\mathrm{MSe}=$ 957.433; $P<0.05]$. This three-way interaction was due to the fact that, on target dimension repetition trials, RTs were faster when the response was repeated rather than changed

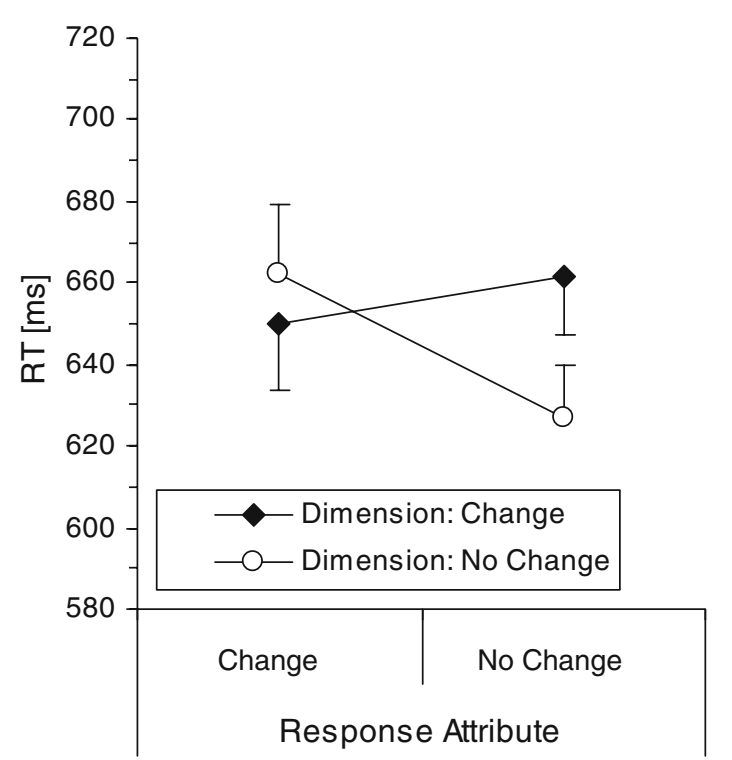

Fig. 3 Experiment 2: Interaction between dimension transition and response transition across trials. RTs were faster to same-dimension, compared to different-dimension, targets on response repetition trials. This pattern (dimension change costs) tended to be reversed on response change trials (dimension change benefit). (Error bars indicate standard error of the mean.)

(626.8 vs. $662.5 \mathrm{~ms}$ ); conversely, on target dimension change trials, RTs were slower when the response was repeated rather than changed (661.6 vs. $649.8 \mathrm{~ms}$ ).

The pattern of interactive intertrial dimension and response change effects is consistent with previous studies [see Müller and Krummenacher (2006), for review; see also Töllner et al. (2008)], who reported RT performance in compound tasks to be contingent on both repetition/change of the target-defining dimension and repetition/change of the response attribute across consecutive trials-despite the fact that the two types of change were uncorrelated. Müller and Krummenacher (2006) [see also Töllner et al. (2008)] suggested that, although the target and response features change independently of each other, the processing systems implicitly expects a link (cf. Kingstone 1992) between target and response attributes such that if the target attribute remains the same, the response (attribute) will remain the same as well; and if the target attributes change, the response (attributed) will be changed as well. Consequently, response selection is expedited in these two cases, compared to the other two cases in which either the target attribute stays the same but the responses change, or the target attribute changes but the response stays the same. In this way, postselective response-related processes (e.g., response selection) may mask preattentive dimension-specific intertrial effects. This was positively demonstrated by Töllner et al. (2008), who found the N2pc (indicative of focal-attentional selection) component to peak earlier and be larger for dimension repetition versus change trials, 


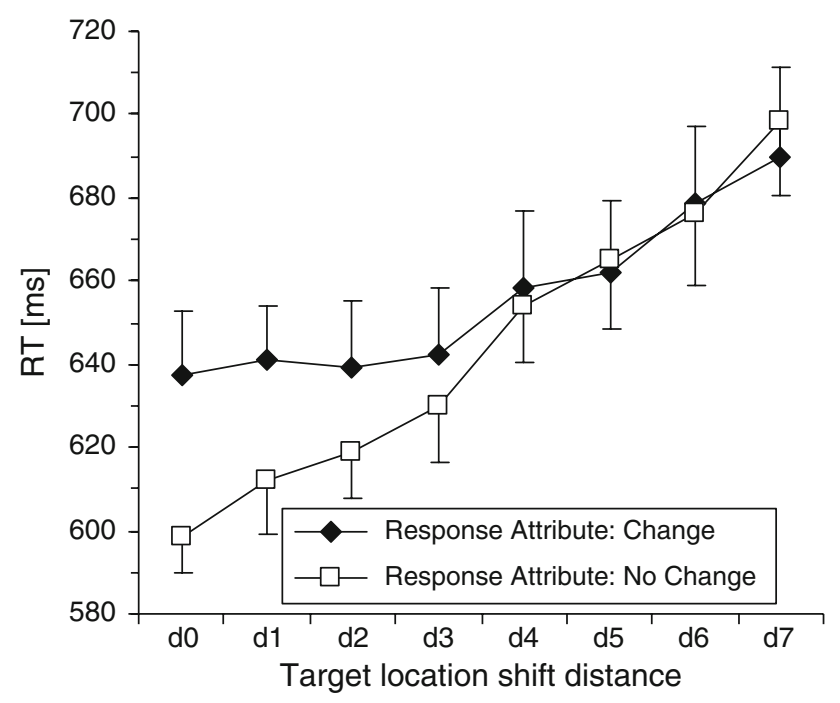

Fig. 4 Experiment 2: Interaction between intertrial response transition and distance of target location shifts. In response repetition trials (white squares), RTs increased linearly with increasing distances between target locations in the current trial $N$ and the previous trial $N-1$. In response change trials (black squares), RTs linearly increase as a function of target location distance, but only for distances $\geq 3$. (Error bars indicate standard error of the mean.)

irrespectively of whether the response was repeated or changed.

In addition to replicating the response transition $x$ dimension transition interaction, Experiment 2 also revealed response transition to interact with intertrial distance between target locations. As can be seen from Fig. 4, when the response attribute remained unchanged, RTs increased linearly by, on average, $14.3 \mathrm{~ms}$ with each unit of distance between target locations on consecutive trials. When the response attribute changed across trials, a comparable increase in $\mathrm{RT}$ with increasing distance was evident for distances $\geq \mathrm{d} 3$, but not for distances $<\mathrm{d} 3$, where the function relating RT to distance was essentially flat. In other words, for such shorter distances $(\mathrm{d} 0, \mathrm{~d} 1, \mathrm{~d} 2)$, there were additional RT costs on response change, relative to repetition, trials. An alternative way of looking at the data is in terms of the slopes of the functions relating RT to distance in the response repetition and change trials, with the (in both cases linear: $R^{2}$ of linear fit, 0.98 vs. 0.89 ) distance effect being more marked on response repetition compared to response change trials (14.1 vs. $7.6 \mathrm{~ms}$ per unit of distance). This suggests a cross-over-type interaction such that, while there is a response change cost at short distances, this tends to reverse into a response change advantage at longer distances. This pattern can be taken to mean that changes of the target location, as well as changes of the target-defining dimension, influence response-related processes: small location shifts (i.e., relatively unchanged locations) tend to bias response-related processing toward selecting an unchanged response (thus delaying the production of a changed response); in contrast, large location shifts induce a bias toward a changed response (retarding production of a repeated response). That is, the linked expectancies between target location repetition/change and response repetition/change can be interpreted along the same lines as those between dimension repetition/change and response/ repetition change-with S-R translations appearing to be affected independently by both types of linkage. ${ }^{1}$

Although interaction between dimension transition and intertrial distance between target locations (see Fig. 2) was nonsignificant $[F(5.231,47.077)=1.619 ; \mathrm{MSe}=1,936.631$; $P=0.171$ (Huyhn-Feldt-corrected $d f$ )], clearly short distances contributed more to the main effect of dimension repetition/change compared to longer distances $[\mathrm{d} 0, \mathrm{~d} 1, \mathrm{~d} 2$ vs. d5, d6, d7: 17.5 vs. $4.1 \mathrm{~ms}$, two-tailed paired-samples $t(9)=3.248, P=0.010]$, suggesting that the dimension repetition advantage diminishes as a function of distance. This is theoretically important, as it is consistent with the notion of a spatial weighting mechanism that operates on the overall-saliency map, weighting the incoming (dimensionspecific feature contrast) signals as a function of their distance from the previous target location.

Overall, the pattern of increasing RT costs associated with increasing shifts of the target location on the current relative to the preceding trial (whether the response was repeated or changed) can be explained by the requirement of spatial-attentional focusing in compound-search tasks introducing a gradient of spatial weighting centered on the target location (the focus of attention) on a given trial, which is then carried over to the next trial. As a result, processing of a target on that trial is delayed the more the further away its location is from that of the preceding target (see 'General discussion').

\footnotetext{
${ }^{1}$ The distance effects appear to be dependent mainly on the spatial separation of the current from the preceding target, where larger distances necessarily involve crossing of the vertical and horizontal field meridians. Further analyses of the distance effects in terms whether they occurred within the same field quadrant, crossed the vertical meridian only, the horizontal meridian only, or both meridians revealed meridian crossing costs (which were unaffected by the number of meridians crossed), consistent with prior studies of the reallocation of attention to an (invalid) target following the presentation of a spatial cue at a nontarget location (e.g., Rizzolatti, Riggio, Dascola \& Umiltà, 1987; Egly \& Homa, 1991). However, it is not clear whether this pattern in the present data reflects a true meridian crossing cost, distinct from a pure distance effect. In an attempt to distinguish between the two types of effect, the data for distance $\mathrm{d} 3$ (i.e., the maximum distance possible within a quadrant; for shorter distances, the data available for analysis were insufficient) were examined for meridian crossing effects. This analysis failed to reveal RTs to new targets presented across one or both field meridians relative to the old target to be longer than RTs to targets presented within the same quadrant. While this suggests a pure distance effect, it cannot be really ruled out that meridian crossing plays a role as well, as even with $\mathrm{d} 3$ there were too few data available to reliably estimate performance in the respective conditions.
} 
Comparison of Experiments 1 and 2

A mixed-design ANOVA comparing RT performance between simple-detection (Experiment 1) and compoundsearch (Experiment 2) tasks confirmed all important differences between the two tasks to be significant. Overall, RTs were faster in Experiment 1 than in Experiment 2 [448.0 vs. $650.0 \mathrm{~ms}$; main effect of experiment, $F(1,18)=89.029$, $\mathrm{MSe}=4,580.828, \quad P<0.001]$. Furthermore, RTs were faster to color compared to orientation targets (435.2 vs. $460.9 \mathrm{~ms}$ ) in Experiment 1, but faster to orientation than to color targets (655.4 vs. $644.5 \mathrm{~ms}$ ) in Experiment 2 [interaction experiment $\times$ dimension, $F(1,18)=25.917 ; \quad \mathrm{MSe}=$ $2,070.585 ; P<0.001]$. Moreover, dimension-based intertrial effects were larger in Experiment 1 relative to Experiment 2 [34.4 vs. $9.9 \mathrm{~ms}$; both effects reliably larger than zero: $t(9)=6.520, P<0.001$, and $t(9)=7.965, P<.001$, respectively; interaction experiment $\times$ dimension transition, $F(1,18)=20.405 ; \mathrm{MSe}=1,178.839 ; P<0.001]$. Conversely, the effect of intertrial target distance was larger in Experiment 2 compared to Experiment 1 [measured in terms of the difference between location repetitions (d0) and the maximum distance shift (d7), the effect was $32.5 \mathrm{~ms}$ in Experiment 1 and $77.8 \mathrm{~ms}$ in Experiment 2; interaction experiment $\times$ intertrial target distance, $F(3.290,59.213)=$ 11.544; $\mathrm{MSe}=1,015.841 ; P<0.001]$. (None of the other two-way and higher-order interactions were significant.) The fact that the intertrial target distance effect was significant in Experiment 1, and the dimensional intertrial transition effect significant in Experiment 2, argues against a qualitative difference in processing between simple detection and compound tasks.

\section{General discussion}

In summary, the interactions between experiment and dimensional intertrial transition and between experiment and intertrial target distance indicate that in (compoundsearch) tasks requiring high focal-attentional resolution, dimension repetition/change effects, although still significant, are considerably reduced compared with (simpledetection) tasks that can be performed with low resolution, and this reduction is accompanied by increased effects of the distance between targets on successive trials.

This pattern is consistent with the idea that, while simple-detection tasks involve largely (though not exclusively) dimension-based weighting, compound-search tasks involve largely (though not exclusively) space-based weighting, with a 'competitive relationship' between both weighting mechanisms - perhaps because both draw on the same limited pool of attentional (weight) resources. An alternative account of this pattern may be derived from
Chan and Hayward's (2008) dual-route account, according to which simple-detection tasks are solved via a nonspatial detection mechanism, which is subject to dimension-based modulations, whereas compound-search (and localization) tasks are solved via a spatial mechanism (the overallsaliency map), which is not subject to dimension-based modulations, but (judging from the present data) may be subject to space-based modulations. Consistent with the dual-route account, there were no statistically significant interactions between dimensional intertrial transition and intertrial target distance in the present experiments, which would suggest that both factors influence separate stages of processing. However, the dual-route account assumes a strict dichotomy between dimension-based and space-based effects, rather than a trade-off relationship — as was revealed in the present study (moreover, there were significant intertrial position effects in the detection task, which cannot occur according to the dual-route model). Given the latter, an account in terms of competition for the same pool of weight resources would appear more plausible. According to this account, space may be considered as just another stimulus dimension, besides color, orientation, etc. (e.g., Nissen 1985; Bundesen 1991), and therefore compete for dimensional (weight) resources when it is task-relevant (but not when it is irrelevant). An alternative view is that the pool of spatial weights is separate from that of dimensional weights, but spatial weighting is invoked only to the extent required by the task, with a general suppressive influence of spatial weighting on the effect of dimensional weighting.

This presupposes that dimension-based effects are really reduced in compound-search (e.g., Experiment 2) relative to simple-detection (Experiment 1) tasks. This may be debatable, as the true dimension-based effect was likely to be obscured in Experiment 2 by implicit S-R linkages along the lines demonstrated by Töllner et al. (2008) [see also Müller and Krummenacher (2006)]. That is, a dimension repetition is associated with an unchanged response, whereas a dimension change is associated with an altered response, delaying S-R translation when the response is changed after a dimension repetition and, respectively, when the response is repeated after a dimension change. This pattern of effects, ${ }^{2}$ which was also evident in the present Experiment 2, makes it difficult to estimate the true dimension repetition/change effect. Judging from the N2pc

\footnotetext{
${ }^{2}$ This pattern of effects is very similar to that found by Hommel (1998) in a prime-probe task, that is, dimension, position, and response repetition effects are large when other aspects also repeat; but they are reduced, absent, or even reversed when another aspect changes. As this pattern is evident across perceptual (dimension and position) and response-related aspects of processing, it is possible that these effects also involve some central processing stage (besides perceptual and response-related stages per se) — such as a stage of 'feature-response binding' assumed in Hommel's (1998) 'event file' theory.
} 
latency difference between dimension repetition and change trials, which Töllner et al. (2008) found to be unaffected by response repetitions/changes, the effect would be relatively small, of the order of $10 \mathrm{~ms}$, compared to the 40- to $30-\mathrm{ms}$ effect typically observed in simple-detection tasks, but again, the N2pc latency effect is itself difficult to interpret in precise temporal terms, because Töllner et al. also found the N2pc amplitude to be enhanced for dimension repetition, compared to change, trials. Nevertheless, even when the smearing of ERP latency differences by amplitude effects is taken into account, the dimensional intertrial effect is likely to be reduced in compound-search relative to simple-detection tasks - consistent with an suppressive influence of space-based weighting (when invoked by the task demands) on the effect of dimension-based weighting.

However, closer inspection of the dimensional intertrial transition $\times$ intertrial target distance interaction in Experiment 2 (although not statistically significant) suggests that this suppression is spatially scaled: as can be seen from Fig. 2, the (significant main) effect of dimension repetition/ change in Experiment 2 was due solely to small distances between target locations on successive trials [d(0) to $d(2)]$, whereas the effect was nonexistent at large distances $[\mathrm{d}(5)$ to $d(7)$ ]. This pattern suggests that dimension-based and space-based weighting processes influence a common processing stage, most likely the overall-saliency map. Nevertheless, space-based weighting may be functionally independent of dimension-based weighting, in that the former may operate via (inhibitory) connections between units within the overall-saliency map and the latter modulating the bottom-up signals to the overall-saliency units. Thus, even if the difference in bottom-up signals between dimension repetition and change trials coming into an overall-saliency unit is itself unaffected by the intertrial target distance (i.e., even if dimension weighting operates equally in parallel across the field), the lower spatial weight assigned to far locations (i.e., weights going towards zero) will reduce the dimension-specific effect as a function of intertrial target distance-assuming that the bottom-up signal is multiplicatively combined with, that is, scaled by the within-map signal — and thereby also increase the time for a target at a changed position to activate the corresponding overall-saliency unit above threshold. [The required multiplicative signal combination may be achieved by an activated saliency unit modulating, via inhibitory links to overall-saliency units within its narrower and wider surround, any bottom-up signals to the surround units (via 'axo-axonal' connections), thereby scaling the dimensionbased input into such units as a function of distance from the focus of attention.]

This account assumes that spatial weighting is positive in sign, with weights ranging from 1 to 0 (decreasing from the focus of attention toward the periphery). Negative weights, indicative of carry-over of inhibition of distractor locations across trials, would lead to the reverse effect of a dimension change benefit, rather than a cost. Note that while inhibitory positional carry-over effects have been observed in singleton feature search tasks (e.g., Maljkovic \& Nakayama 1996), Geyer and Müller (2008b) showed that inhibition of previous distractor locations is obtained only with small numbers of regularly arranged display items, whereas facilitation for previous target locations is obtained independently of the item number and arrangement. Thus, extrapolating from the findings of Geyer and Müller, the assumption of a gradient of facilitatory space-based weighting (declining as a function of distance from the focus of attention) would appear tenable.

In summary, the present data reveal a strong component of spatial weighting, with carry-over of weights across trials, in (compound-search) tasks that require focal attention for response. Space-based weighting appears to suppress the effect of dimensional weighting on signal integration by units in the overall-saliency map, with the suppressive effect being scaled according to the distance of a given location from the focus of attention. In contrast, in simpledetection tasks, dimension-based effects may be expressed almost fully, with near-equal and -maximal spatial weights assigned to locations across the display. While there seems to be a suppressive influence of spatial on dimensional weighting, the precise spatial-weighting function and the details of the interaction of spatial with dimensional weighting signals need to be worked out in further studies.

Acknowledgments The present study was supported by Swiss National Science Foundation (SNSF) grant PP001-110543/1 (J. Krummenacher) and German National Science Foundation (DFG) grant FOR480 (J. Krummenacher, H. J. Müller, and T. Geyer).

\section{References}

Bundesen, C. (1991). Visual selection of features and objects: Is location special? A reinterpretation of Nissen's (1985) findings. Perception and Psychophysics, 50, 87-89.

Cave, K. R., \& Wolfe, J. M. (1990). Modeling the role of parallel processing in visual search. Cognitive Psychology, 22, 225-271.

Chan, L. K. H., \& Hayward, W. G. (2008). Feature integration theory revisited: Dissociating feature detection and attentional guidance in visual search. Journal of Experimental Psychology: Human Perception \& Performance (in press).

Cohen, A., \& Magen, H. (1999). Intra- and cross-dimensional visual search for single feature targets. Perception and Psychophysics, 61, 291-307.

Egly, R., \& Homa, D. (1991). Reallocation of visual attention. Journal of Experimental Psychology: Human Perception and Performance, 17, 142-159.

Eimer, M. (1996). The N2pc component as an indicator of attentional selectivity. Electroencephalography and Clinical Neurophysiology, 99, 225-234.

Folk, C. L., Remington, R. W., \& Johnston, I. C. (1992). Involuntary covert orienting is contingent on attentional control settings. 
Journal of Experimental Psychology: Human Perception and Performance, 18, 1030-1044.

Found, A., \& Müller, H. J. (1996). Searching for unknown feature targets on more than one dimension: Investigating a "dimensionweighting" account. Perception and Psychophysics, 58, 88-101.

Geyer, T., \& Müller, H. J. (2008a). Distinct, but top-down modulable color and positional priming mechanisms in visual pop-out search. Psychological Research (this issue).

Geyer, T., \& Müller, H. J. (2008b). Positional priming of pop-out: A relational-encoding account. Journal of Experimental Psychology: Human Perception \& Performance (submitted).

Geyer, T., Müller, H. J., \& Krummenacher, J. (2007). Cross-trial priming of element positions in pop-out visual search is dependent on regular stimulus arrangement. Journal of Experimental Psychology: Human Perception and Performance, 33, 788-797.

Hommel, B. (1998). Event files: Evidence for automatic integration of stimulus-response episodes. Visual Cognition, 5, 183-216.

Hopf, J. M., Boelmans, K., Schoenfeld, A. M., Heinze, H. J., \& Luck, S. J. (2002). How does attention attenuate target-distractor interference in vision? Evidence from magnetoencephalographic recordings. Cognitive Brain Research, 15, 17-29.

Kingstone, A. (1992). Combining expectancies. Quarterly Journal Experimental Psychology, 44A, 69-104.

Krummenacher, J., Müller, H. J., \& Heller, D. (2002a). Visual search for dimensionally redundant pop-out targets: Parallel-coactive processing of dimensions is location-specific. Journal of Experimental Psychology: Human Perception and Performance, 28, 1303-1322.

Krummenacher, J., Müller, H. J., \& Heller, D. (2002b). Visual search for dimensionally redundant pop-out targets: Redundancy gains in compound tasks. Visual Cognition, 9, 801-837.

Maljkovic, V., \& Nakayama, K. (1996). Priming of popout: II. Role of position. Perception and Psychophysics, 58, 977-991.

Mortier, K., Theeuwes, J., \& Starreveld, P. (2005). Response selection modulates visual search within and across dimensions. Journal of Experimental Psychology: Human Perception and Performance, $31,542-557$.

Müller, H. J., Heller, D., \& Ziegler, J. (1995). Visual search for singleton feature targets within and across feature dimensions. Perception and Psychophysics, 57, 1-17.

Müller, H. J., \& Krummenacher, J. (2006). Locus of dimension weighting: Pre-attentive or post-selective? Visual Cognition, 14, 490-513.

Müller, H. J., Reimann, B., \& Krummenacher, J. (2003). Visual search for singleton feature targets across dimensions: Stimulus- and expectancy-driven effects in dimensional weighting. Journal of Experimental Psychology: Human Perception and Performance, 29, 1021-1035.

Nissen, M. J. (1985). Accessing features and objects: Is location special? In M. I. Posner \& O. S. M. Marin (Eds.), Attention and Performance XI (pp. 205-219). Hillsdale: Erlbaum.

Pollmann, S., Weidner, R., Müller, H. J., \& von Cramon, D. Y. (2000). A fronto-posterior network involved in visual dimension changes. Journal of Cognitive Neuroscience, 12, 480-494.

Pollmann, S., Weidner, R., Müller, H. J., \& von Cramon, D. Y. (2006). Neural correlates of visual dimension weighting. Visual Cognition, 14, 877-897.

Rizzolatti, G., Riggio, L., Dascola, I., \& Umiltà, C. (1987). Reorienting attention across the horizontal and vertical meridians: Evidence in favor of a premotor theory of attention. Neuropsychologia, 25, $31-40$.

Theeuwes, J., Reimann, B., \& Mortier, K. (2006). Visual search for featural singletons: No top-down modulation, only bottom-up priming. Visual Cognition, 14, 466-489.

Töllner, T., Gramann, K., Müller, H. J., Kiss, M., \& Eimer, M. (2008). Electrophysiological markers of visual dimension changes and response changes. Journal of Experimental Psychology: Human Perception and Performance, 34, 531-542.

Treisman, A. (1988). Features and objects: The fourteenth Bartlett memorial lectures. Quarterly Journal of Experimental Psychology, 40A, 201-237.

Treisman, A., \& Gelade, G. (1980). A feature integration theory of attention. Cognitive Psychology, 12, 97-136.

Treisman, A., \& Sato, S. (1990). Conjunction search revisited. Journal of Experimental Psychology: Human Perception and Performance, 16, 459-478.

Wolfe, J. M. (1994). Guided search 2.0: A revised model of visual search. Psychonomic Bulletin and Review, 1, 202-238.

Wolfe, J. M., Cave, K. R., \& Franzel, S. L. (1989). Guided search: An alternative to the feature integration model for visual search. Journal of Experimental Psychology: Human Perception and Performance, 15, 419-433.

Woodman, G. F., \& Luck, S. J. (1999). Electrophysiological measurement of rapid shifts of attention during visual search. Nature, 400 , 867-869.

Zehetleitner, M., \& Müller, H. J. (2008). A mathematical model of the dimension weighting account. Ludwig Maximilian University Munich (unpublished manuscript). 